This material has been provided by the publisher for your convenience. It may not be further reproduced in any manner, including (but not limited to) reprinting, photocopying, electronic storage or transmission, or uploading onto the Internet. It may not be redistributed, amended, or overprinted. Reproduction of this material without permission of the publisher violates federal law and is punishable under Title 17 of the United States Code (Copyright Act) and various international treaties. Reprints or permission to reprint may be ordered by contacting dfagen@avma.org .

\title{
Theriogenology Question of the Month
}

\section{In cooperation with}

\section{History}

A black 2-year-old nulliparous female Suri alpaca was evaluated in July at the Kansas State University Veterinary Medical Teaching Hospital because of infertility. The alpaca had been mated multiple times to a fertile male during the preceding spring. Matings were as anticipated, and each lasted approximately 15 minutes. The alpaca was not sexually receptive to the male the week after the matings, but was sexually receptive again 1 week later ( 2 weeks after the matings).

Initial evaluation revealed that the alpaca was bright, alert, and responsive. Findings of physical examination were within anticipated limits. Transrectal ultrasonography revealed a large (approx $10 \mathrm{~cm}$ in

This report was submitted by Maria S. Ferrer, DVM, MS, DACT; David E. Anderson, DVM, MS, DACVS; Meredyth L. Jones, DVM, MS, DACVIM; Matt D. Miesner, DVM, MS, DACVIM; Terje Raudsepp, PhD; Felipe Avila, BSc; and Michelle A. Kutzler, DVM, PhD, DACT; from the Department of Veterinary Clinical Sciences, College of Veterinary Medicine, Kansas State University, Manhattan, KS 66506 (Ferrer, Anderson, Jones, Miesner); the Department of Veterinary Integrative Biosciences, College of Veterinary Medicine, Texas A\&M University, College Station, TX 77843 (Raudsepp, Avila); and the Department of Animal Sciences, College of Agricultural Sciences, Oregon State University, Corvallis, OR 97331 (Kutzler). Dr. Anderson's present address is Department of Large Animal Sciences, College of Veterinary Medicine, University of Tennessee, Knoxville, TN 37996. Dr. Jones' present address is Department of Large Animal Clinical Sciences, Texas A\&M University, College Station, TX 77843.

The authors declare that there is no conflict of interest that could be perceived as prejudicing the impartiality of the information reported. Address correspondence to Dr. Ferrer (mferrer@vet.k-state.edu). diameter) fluid-filled uterine horn. The fluid was anechoic and contained a few free-floating hyperechoic particles; however, the ventral border of the uterine horn could not be seen during transrectal ultrasonography. Transabdominal ultrasonography was performed, which confirmed the presence of a fluid-filled uterine horn on the left side of the abdomen. The uterus extended to the midventral aspect of the abdomen. No fetal structures were identified within the uterus. A second uterine horn and the ovaries were not detected during transrectal or transabdominal ultrasonography.

Direct visual vaginal examination was performed with a speculum, which revealed no anatomic abnormalities of the vestibule, vagina, or cervix. Vaginoscopy and hysteroscopy were performed with a flexible endoscope. The vagina had a normal appearance, the cervix was open, and no discharge was observed. The uterine lumen consisted of a single tubular structure with no evidence of free fluid. A congenital anatomic abnormality was suspected because no communication to a second uterine horn could be found. To rule out a chromosomal abnormality, whole blood was collected into a heparinized tube and submitted for chromosome analysis. ${ }^{a}$ The karyotype was $74, \mathrm{XX}$, and a PCR test for the SRY gene yielded negative results, which were consistent with a genetically normal female alpaca.

\section{Question}

What congenital abnormality should be suspected in this alpaca? Please turn the page. 


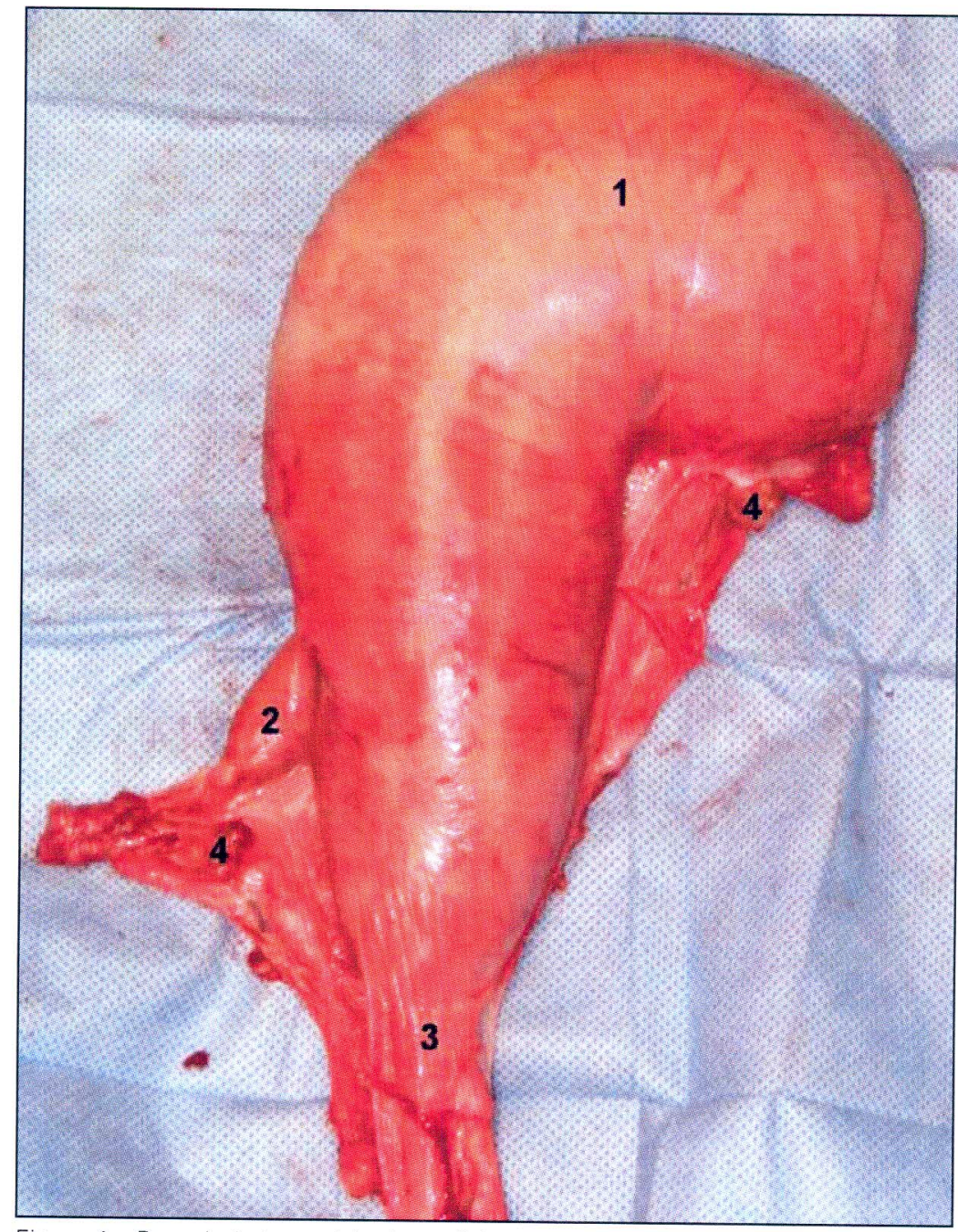

Figure 1-Dorsal photographic view of the genital tract of the alpaca during necropsy. The right uterine horn (1) is a large, thin-walled, and fluid-filled structure. The left uterine horn (2), cervix (3), and both ovaries (4) are within anticipated limits.

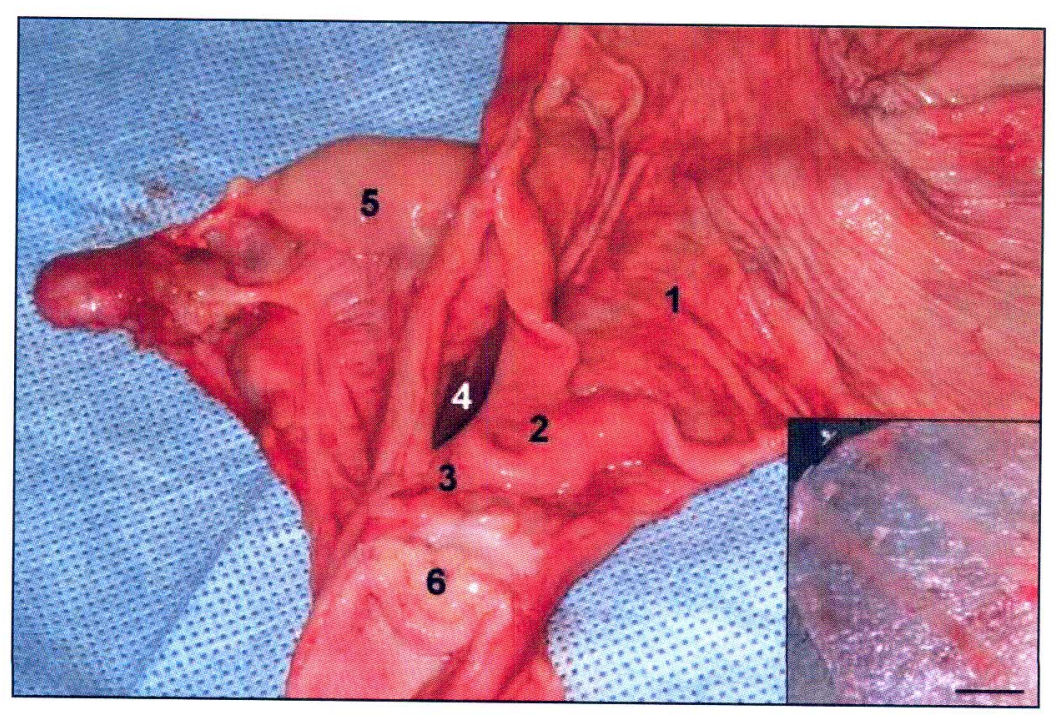

\section{Answer}

Segmental aplasia of the uterine horn with mucometra.

\section{Results}

The owner declined further diagnostic testing or treatment options and elected to have the alpaca euthanized because of a poor prognosis for fertility, the possibility of a genetic cause for the condition, and concerns about the animal's well-being. During necropsy, both ovaries were found to have follicular activity and were anatomically normal. The left ovary contained 3 large follicles (each approx 6 to $8 \mathrm{~mm}$ in diameter) and multiple medium follicles (each approx $4 \mathrm{~mm}$ in diameter), and the right ovary contained multiple small follicles (each approx $3 \mathrm{~mm}$ in diameter). The left uterine horn was within anticipated limits, but the right uterine horn was a grossly large, fluid-filled, and thin-walled structure (Figure 1). The right uterine horn was approximately 2.5 to 3 times as large in diameter and 5 times as long as the left uterine horn. The caudal portion of the right uterine horn ended in a blind pouch, and no communication with the uterine body or cervix could be identified (Figure 2). The surface of the endometrium was smooth with no visible endometrial folds. There was a large amount (approx $600 \mathrm{~mL}$ ) of cloudy mucoid material within the lumen of the right uterine horn.

A uterine torsion or displacement of the uterus to the left side of the abdomen was not identified during necropsy. However, temporary displacement of the uterus by the gastrointestinal tract or bladder or as a result of the weight of the fluid at the time of the ultrasonographic examination could not be ruled out and may explain the reason that the fluidfilled right uterine horn was visible during transabdominal ultrasonography via a left approach.

Cytologic examination of uterine contents was conducted. Slides were stained with a modified Wright stain.

Figure 2-Dorsal photographic view of the genital tract of the alpaca in Figure 1 after the dorsal wall of the vagina, cervix, uterine body, and right uterine horn were incised to expose the lumen. The right uterine horn (1) ends in a blind pouch (2), and there is no communication with the uterine body (3). The scalpel blade (4) is in the lumen of the left uterine horn (5), which is contiguous with the uterine body (3) and cervix (6). Inset-Photograph of the wall of the right uterine horn. Notice that the wall is so thin that it appears to be transparent or translucent in some regions. $\mathrm{Bar}=20 \mathrm{~mm}$. 
Evaluation via oil immersion with a light microscope revealed a moderate amount of RBCs and only a few lymphocytes, macrophages, and neutrophils in $20 \mathrm{hpf}$ (100X magnification).

Formalin-fixed tissue from the uterine wall from both uterine horns and the septum was submitted for histologic examination. The septum consisted of a central portion of myometrium, with endometrial tissue on both sides. The wall of the left uterine horn was approximately $6 \mathrm{~mm}$ thick. The wall of the right uterine horn was 1 to $3 \mathrm{~mm}$ thick. The endometrial epithelium of the right uterine horn was atrophied. There was diffuse inflammation of the lamina propria of the endometrium in both uterine horns, with lymphocytes, neutrophils, and plasma cells infiltrating the endometrium. The diagnosis was endometritis with endometrial atrophy of the right uterine horn.

\section{Discussion}

Differential diagnoses for a large fluid-filled uterus include pregnancy, hydrometra, mucometra, and pyometra. Pregnancy was ruled out in the alpaca reported here on the basis of a failure to identify fetal structures during ultrasonography; therefore, hydrometra, mucometra, or pyometra was suspected. These conditions develop in camelids secondary to retention of uterine contents caused by obstruction of the uterine lumen, cervix, or vagina. ${ }^{1,2}$ Obstruction can be caused by scar tissue or adhesions, prolonged progesterone treatment, or congenital defects. ${ }^{1,2}$ Congenital abnormalities should be considered as a cause of fluid accumulation in nulliparous females. Given the noninflammatory nature and characteristics of the fluid, a diagnosis of mucometra was made in the alpaca of the present report.

In a developing embryo, the paramesonephric ducts originate from invaginations of the coelomic epithelium of the urogenital ridges lateral to the mesonephros. These paired structures of mesodermic origin course in a cranial to caudal direction, laterally initially and then medially over the mesonephric ducts to fuse caudally with the urogenital sinus. A solid cord develops first, and then a lumen forms within the cord. In a female fetus, in the absence of Müllerian-inhibiting factor produced by Sertoli cells, the paramesonephric ducts are retained and differentiate into the oviducts, uterus, cervix, and cranial portion of the vagina. Segmental aplasia is a focal defect in the development of the paramesonephric ducts. ${ }^{3}$ In the most severe cases of complete aplasia involving the uterus, an entire uterine horn may be missing. In milder cases of partial aplasia, there is a failure of lumen development in a portion of the uterine horn. ${ }^{3}$ The caudal portion of the right paramesonephric duct is commonly affected in most species, except sheep. ${ }^{4-7}$ The reason for differential involvement of 1 side may be differences in genetic control or synchrony of development of the right and left uterine horns. ${ }^{3}$ In cows, segmental aplasia is a sex-limited (female-associated) autosomal recessive trait linked to a white coat in the Shorthorn and Belgian Blue breeds. The condition is known as white heifer disease. ${ }^{3}$ In goats, segmental aplasia has also been linked to the polled gene. ${ }^{7}$ It is unknown whether there is a genetic cause of segmental aplasia in alpacas and whether the underlying genetic mechanisms are similar to those described in other species. In the present report, the condition was found in a black (pigmented) alpaca, but in cows, it is linked to a depigmentation phenotype. A family history of this alpaca with regard to congenital abnormalities was not available.

Segmental aplasia of the paramesonephric ducts affecting the uterus has been reported in cows, ${ }^{3}$ dogs, ${ }^{4}$ cats,${ }^{4}$ ferrets, ${ }^{5}$ sheep, ${ }^{6}$ and goats. ${ }^{7}$ Affected females are chromosomally normal and typically have physiologically normal ovarian development and activity. ${ }^{5}$ The portions cranial to the missing segment often contain mucoid, watery, serosanguineous, or inspissated material from endometrial gland secretions. ${ }^{3-7}$ Females may be infertile or anestrous or may have signs of abdominal discomfort. ${ }^{3-7}$ Uterine rupture secondary to overdistention and subsequent peritonitis is a possible complication. ${ }^{3,7}$ Segmental aplasia of the uterine horn was suspected on the basis of ultrasonographic findings and confirmed via laparoscopy in 1 alpaca and 1 camel. However, no further descriptions of the medical history, findings, and outcome were provided in that report. The findings in the alpaca of the present report were consistent with those in other species, with the caudal segment of the right uterine horn affected. The remainder of the genital tract had no gross abnormalities, both ovaries had follicular activity, and the female had a genotypically normal karyotype. In other reports, ${ }^{3-7}$ only a band of connective tissue without portions of uterine wall separated the 2 segments of the uterus. In the alpaca of the present report, a core of myometrium with uterine mucosa on both sides formed the septum. Partial segmental aplasia of the mucosa with normalappearing myometrium has been reported in a cow. ${ }^{8}$

Atrophy of the endometrium was evident grossly on histologic examination. This was thought to be secondary to pressure caused by the accumulated fluid. The behavioral history of the alpaca was consistent with ovulation with fertilization failure or early pregnancy loss prior to maternal recognition of pregnancy. Atrophy of the endometrium may result in lack of release of prostaglandin $\mathrm{F}_{2 \alpha}$ from the affected uterine horn. Given the local countercurrent mechanism involved in luteolysis in ruminants, this may lead to persistent anestrus as a result of lack of luteolysis of the corpus luteum on the ovary ipsilateral to the gravid uterine horn. ${ }^{3}$ In camelids, the right uterine vein drains both the right and left uterine horns, whereas the left uterine vein drains only the left uterine horn. This crossover from the left to the right side appears to explain the luteolytic effect for the left uterine horn over both the left and right ovaries via a local utero-ovarian countercurrent mechanism. Because the left uterine horn was not affected in the alpaca of the present report, there was luteolysis and a return to sexual receptivity, irrespective of the side of ovulation.

The wall of the right uterine horn was extremely thin. If the condition were not treated, additional fluid accumulation could have resulted in uterine rupture. Ovariohysterectomy or partial hysterectomy may have been potential surgical options.

\section{Outcome}

The alpaca was euthanized because of a poor prognosis for fertility, the possibility of a genetic cause for the 
condition, and the owner's concern about the animal's well-being. Possible treatment for segmental aplasia includes ovariohysterectomy or partial hysterectomy if an owner wants to keep the female as a companion animal.

a. Molecular Cytogenetics and Genomics Laboratory, Department of Veterinary Integrative Biosciences, College of Veterinary Medicine and Biomedical Sciences, Texas A\&M University, College Station, Tex.

\section{References}

1. Tibary A, Anouassi A, Memon MA. Approach to diagnosis of infertility in camelids: retrospective study in alpaca, llamas and camels. J Camel Pract Res 2001;8:167-179.

2. Tan RH, Dascanio JJ. Infertility associated with persistent hymen in an alpaca and a llama. Can Vet J 2008;49:1113-1117.
3. Riley CB, Kulik K, Crane M, et al. Management of infertility due to unilateral segmental aplasia of the paramesonephric (Müllerian) duct in Holstein-Friesian cattle-a case-based review and update. Bovine Pract 2007;41:24-31.

4. McIntyre RL, Levy JK, Roberts JF, et al. Developmental uterine anomalies in cats and dogs undergoing elective ovariohysterectomy. J Am Vet Med Assoc 2010;237:542-546.

5. Batista-Arteaga M, Alamo D, Herráez P, et al. Segmental atresia of the uterus associated with hydrometra in a ferret. Vet Rec 2007;161:759-760.

6. Smith KC, Long SE, Parkinson TJ. Congenital abnormalities of the ovine paramesonephric ducts. Br Vet J 1995;151:443-452.

7. Webb P. Segmental aplasia and hydrometra in a goat. Vet Rec 1985;117:3.

8. Peterson JE, Parsonson IM, Newsam IDB, et al. Infertility in dairy heifers with particular reference to a high incidence of developmental defects of the paramesonephric ducts. Aust Vet $J$ $1966: 42: 430-436$ 